\title{
Distribution Curve of Three-dimensional Space of Using Frequency Based on the Interface of Java3D and MySQL Database
}

\author{
Mei Xu \\ Qujing Normal University, Qujing, 655011, China \\ xu_mei2014@yeah.net
}

Keywords: MySQL database, 3D distribution, Indicator, Mandarin, Visualization, Java3D.

\begin{abstract}
The difference of national minority has not been in-depth comparative study by circles in the oral English grammar, in which the differences of indicator markers are more prominent. In order to research on the problem of using minority mandarin indication mark, this paper designs the use frequency of indication mark word MySQL database based on the collection of language use data, and establishes the interface with database using Java3D, and the rendering function of using Java3D calculates the characteristic of using indication mark word. Using 3D visual rendering algorithm, this paper designs the 3D visualization mathematical model and evolution equation, and works out the Java3D program. Through the simulation calculation, we can obtain the threedimensional space distribution curve of minority indication mark word usage characteristic, which improves data reference for the study of minority mandarin.
\end{abstract}

\section{Introduction}

With the rapid development of computer simulation visualization, more and more visualization tools are emerged. As the Java language, Java 3D are sought after by people in the expansion of the direction [1,2]. However, the current research mostly focuses on the discussion of Java 3D scene graph, namely the overall layout and flow visualization of virtual scene, and it is rarely involved in the realization process and details. In this paper, the visualization functions of using Java3D are analyzed and studied on the characteristics of minority languages, to design the indicator database [3-5]. Using Java3D software carries out calculation on the characteristics of demonstrative pronouns, in which the overall framework is shown in Figure 1.

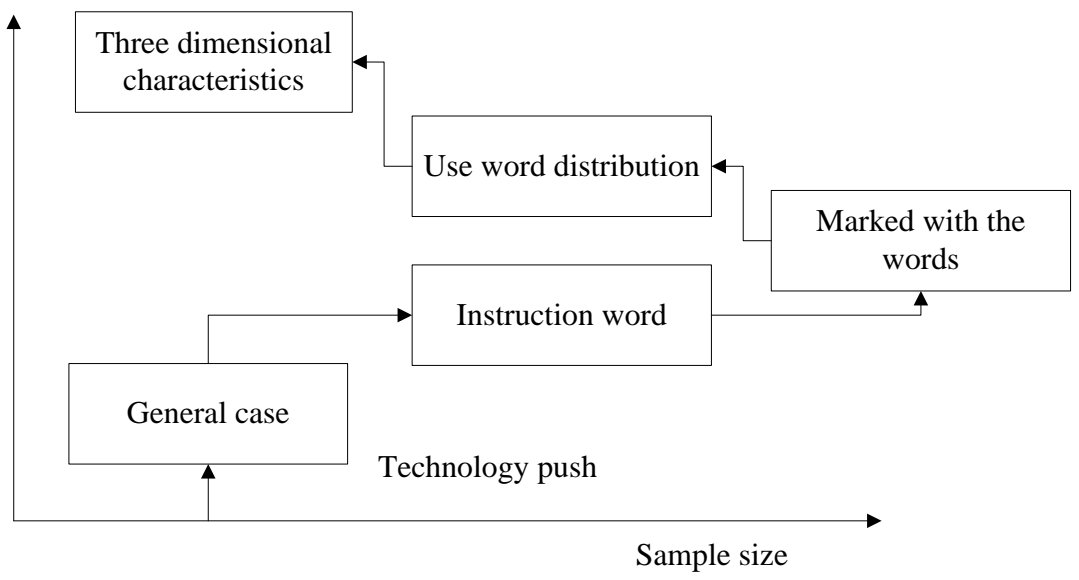

Fig.1: The overall design framework of the indication marker statistical calculation

Figure 1 shows the overall framework of indicating mark statistical calculation designed in this paper, it can be seen from the chart that the framework takes sample size and word capacity as the fundamental basis [6]. Through the general case, indicating words and mark words to calculate the frequency of use, we can obtain three-dimensional space distribution characteristics of the word, which improves data support for the study of minority mandarin language features. 


\section{Design of Mandarin Indication Mark Database Algorithm}

In order to research on the problem of using minority mandarin indication mark time, this paper designs MySQL database by using data language collection, using Java3D has built links with database, and the rendering function of using Java3D has calculated the use characteristic of indication mark word $[7,8]$. In order to use the characteristic of statistical indicating mark word, we choose that, this, in that and in this as regional rendering, the definition is equal to the number of the grid nodes vertices as shown in Figure 2.

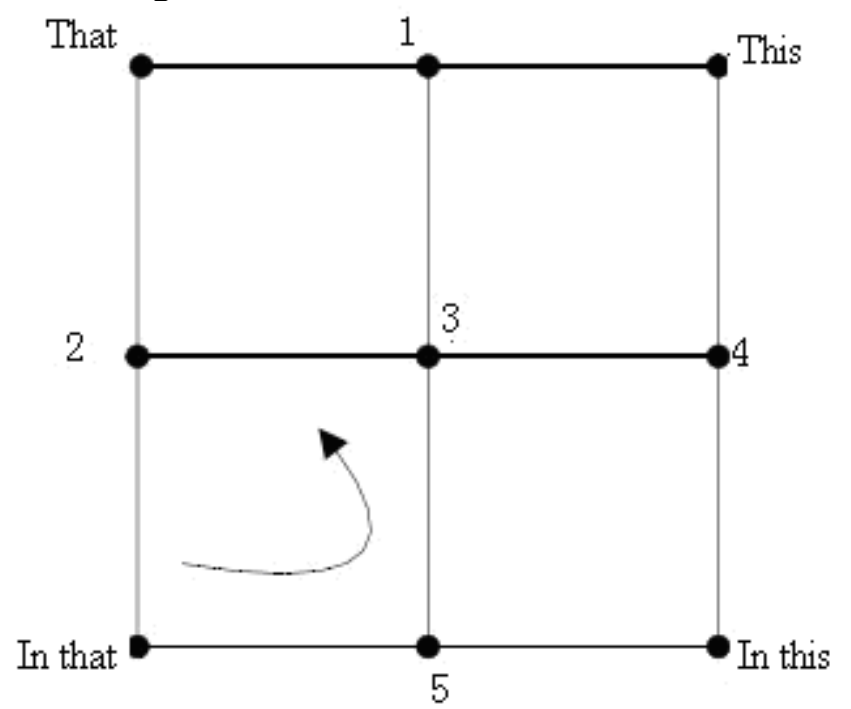

Fig.2: Schematic diagram of regional rendering quadrilateral method

First of all, the use of constructor can construct a polygonal region object, there are 9 peak area, and the grid array of rendering the $3 * 3$ vertices need to draw $2 * 2$ rectangles, the relationship between the three are continuous [9-11]. Assume that these three effects can use $M, N$ and $V$ to express, finally the visualization model can be established

$$
\begin{aligned}
& W=\max [\min (a M, b N, c V)],(a, b, c>0) \\
& \text { s.t. }: M<M_{j}, N<N_{j}, V<V_{j}
\end{aligned}
$$

Among then, $a, b$ and $c$ show node parameters; $M_{j}, N_{j}$ and $V_{j}$ show node projection. So, if there are

$$
\begin{aligned}
& a M>c V \text {, and } b N>c V \text {, then } W=\max (c V) ; \\
& a M>b N \text {, and } c V>b N \text {, then } W=\max (b N) ; \\
& b N>a M, \text { and } c V>a M \text {, then } W=\max (a M) ;
\end{aligned}
$$

Hypothesis that $d 1, d 2$ and $d 3$ indicate the relationship between he change rate and the original state, its evolution equation is

$$
\begin{aligned}
& d M / d t=d 1 M+a(M, N, V) ; \\
& d N / d t=d 2 M+b(M, N, V) ; \\
& d V / d t=d 3 M+c(M, N, V)
\end{aligned}
$$

The nonlinear relationship formula used by indicating mark is

$$
d W / d t=(d+j) W-a W^{3}+W 1 .
$$

The form of three dimensional complex variable functions can be expressed as

$$
W(j)=\frac{-(d+j)}{2} W^{2}+\frac{a}{4} W^{4} .
$$

In order to realize above rendering algorithm, we can achieve the purposes of indication mark space visualization display [12]. Using Java3D language carries out programming for the algorithm, the main procedures are as follows: 
The model can achieve the cache strategy of ST training content center network, and the programming form is as follow:

line=LineStripArray （stripvertexCount）

line.setCoordinate(0,coordinate_V0)

line.setWord(0,word_0)

line.setCoordiante(1,coordinate_V1)

line.setWord(1,word_1)

line.setCoordiante(2,coordinate_V1)

line.setWord(2,word_1)

line.setCoordiante(3,coordinate_V2)

line.setWord(3,word_2)

line.setCoordinate(716,coordinate_V358)

line.setWord(716,coordinate_358)

line.setCoordiante(717,coordinate_V359)

line.setWord(717,word_359)

\section{Statistical Analysis of the Minority Mandarin Indication Mark Database}

In order to verify the effectiveness and reliability of3D visualization mathematical model and its evolution equation designed in section second, this paper designs the minority mandarin indicating mark word database, to analyze the database samples of using Java3D software, in which the import process data in a database is shown in Figure 3.

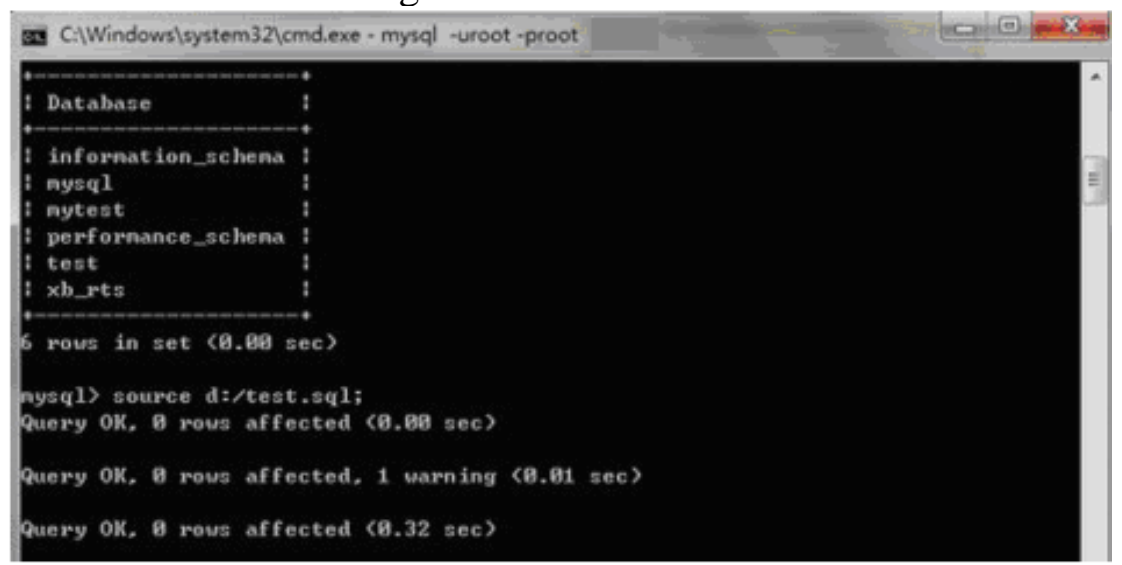

Fig. 3 Indicating mark word data import database

Java 3D is a Java language extension in the three-dimensional graphics field, which is a set of application programming interface (API) [13]. Using the Java 3D program, programmers only need call the API programming, and the clients need only use standard Java virtual machine that can be browsed, therefore there are the advantages of no need to install the plug-in.

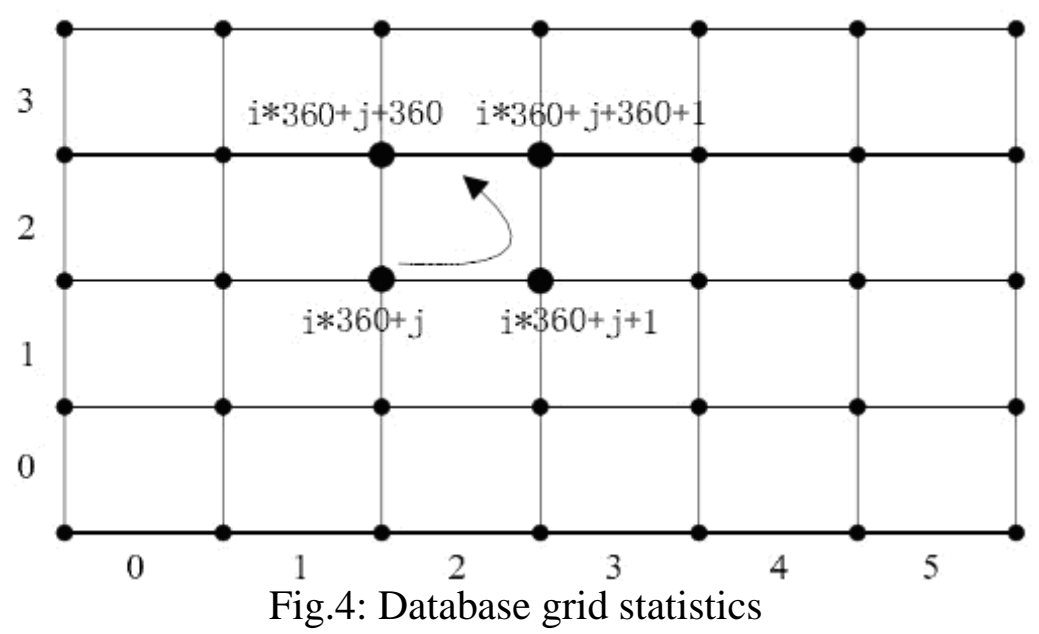


As shown in Figure 4, the Java 3D can only render a basic unit. For each grid, making the lower left corner node is 0 , and then the other three nodes are respectively 1,2 and 3 according to counterclockwise order [14]. In the $181 * 360$ vertices, they is the serial number that is $i * 360+j$, the visualization of the results are obtained by calculating as shown in Figure 5.

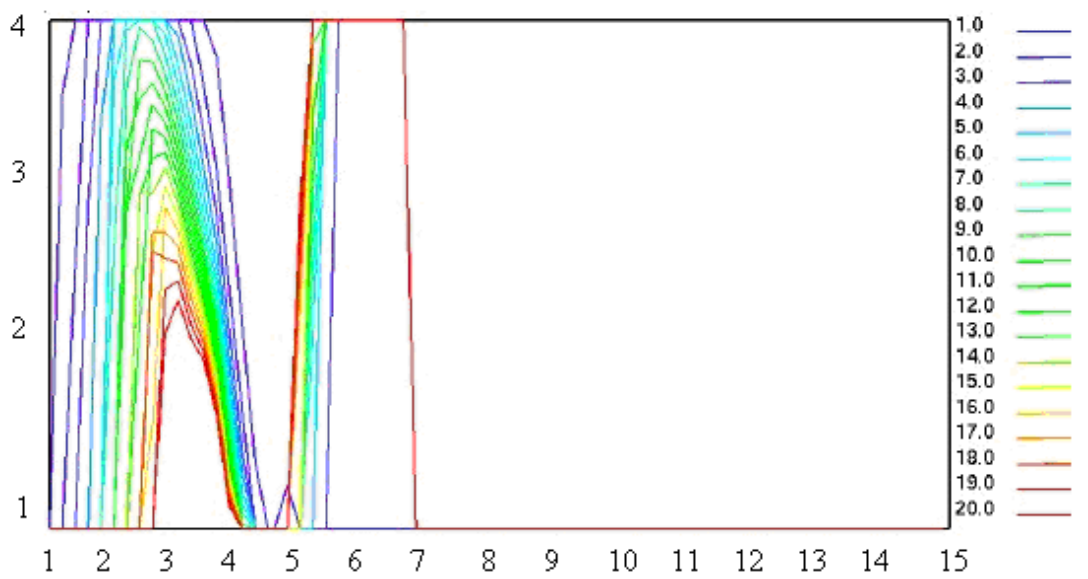

Fig.5: The frequency of indicates mark distribution line

The longitudinal coordinates of 1,2 , 3and 4 are respectively this, in this, that and in that word, and the abscissa represents the database sample size, different colors represent different frequency of use. It can be seen from the chart that the frequency of different word use has obviously samples capacity distribution, in which the distribution is the most obvious within the scope of low sample capacity.

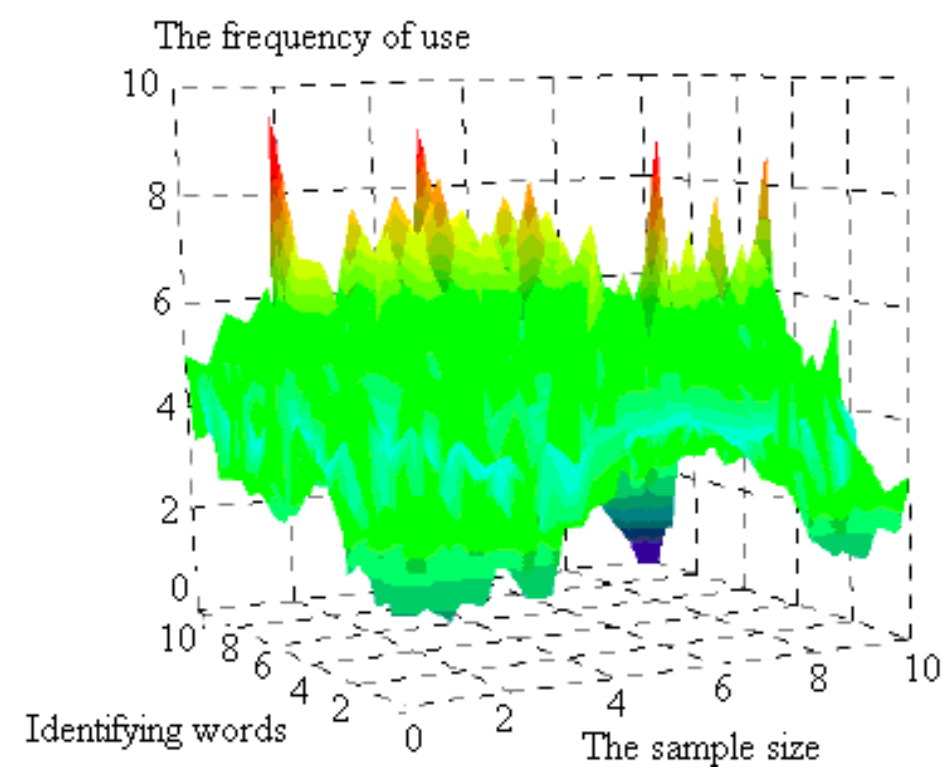

Fig.6: The three dimensional characteristics of indicating mark word database

Figure 6 shows the spatial characteristics of the indication mark words counted calculation by using Java3D, and then we can obtain the distribution curve of three-dimensional space. In order to further reveal the use frequency of each indication mark word distribution, this, in this, that and in that are respectively drawn the distribution curves as shown in Figure 7. 


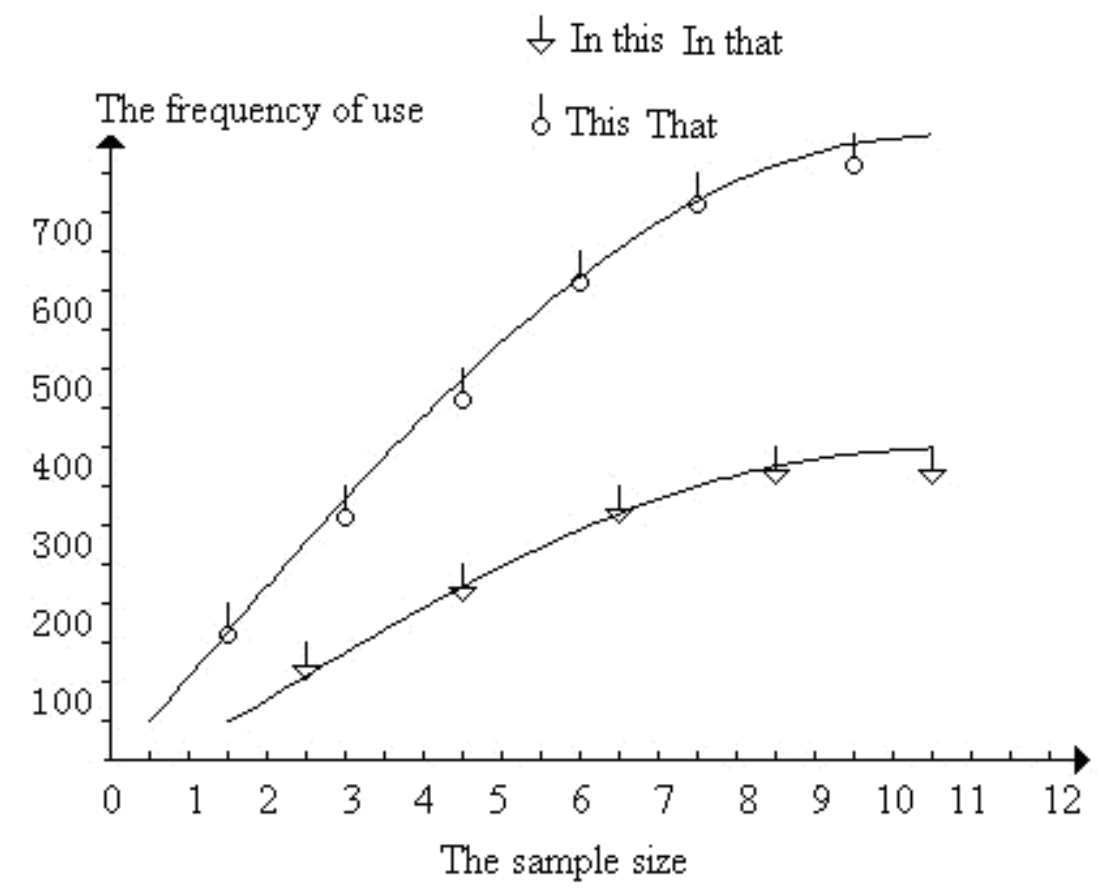

Fig.7: The use frequency of indication marker overall distribution

The overall use frequency of this, that, in this and in that carry out statistical analysis, using Java3D respectively draw their variation curve of the sample volume rendering in the frequency of use [15]. It can be seen from the chart that the frequency of this and that use are higher, and then referring to the cases and mark cases are been statistics, its results has been shown in Table 1 .

Table 1: The frequency of indication mark

\begin{tabular}{llll}
\hline The use of words & General case & Refer to the case & Mark case \\
\hline That & 688 & 233 & 456 \\
In that & 325 & 125 & 366 \\
This & 412 & 108 & 236 \\
In this & 311 & 98 & 121 \\
\hline
\end{tabular}

In the indication mark frequency of the minority mandarin, that and this are higher than in that and in this, and there are also these trend in the mean generation cases and mark case, indication mark word occurrence frequency has the vital significance for the study of spoken language, especially the study of ordinary words in the minority nationalities. We can exit the basic structure of mandarin language by using data, to reveal the local language features.

\section{Summary}

(1) Based on the 3D visualization rendering algorithm, this paper designs the visualization mathematical model and evolution equation of indication mark word statistical analysis and using Java3D software carries out programming design for the algorithm, to establish the Java3D and MySQL database interface.

(2) Using the 3D rendering functions in Java3D, the regional grid of indication mark word is as a unit, to calculate the indicating case and mark case of indication mark word. Finally, we can obtain the three-dimensional space distribution curve of minority indication mark word usage characteristic, which provides a new computer method for the study of minority language feature.

\section{References}

[1] W.B. Yang. The programming and application of the access database in VB. Journal of Chifeng University, 2011(9): 61-65. 
[2] L.L. Jiang, W.W. Jiang. Access database and SQL Server database. Knowledge economy, 2010(4): 34-36.

[3] Y. Hu. Design of librarians learning platform. Knowledge economy, 2010(16): 65-68.

[4] X.N. Zhang, X.L. Zhang. Application of Acess backup and restore database. Fujian computer, 2011(9): 56-59.

[5] Y. Zheng. Construction of talking about blog exchange platform. Chinese medical education technology, 2011(3)

[6] L.Q. Pang. Combined with ACESS system application and its internal control. Modern business, 2008(11):96-99.

[7] P.Y. Yang, P.Q. Jin, L.H. Yue. A time sensitive SSD and HDD efficient hybrid storage model. Journal of computer, 2012(11): 67-79.

[8] S.B. Dou, L.H. Yang, W.H. Gong. An effective heterogeneous disk high efficiency cache mechanism. Computer system application, 2011(11): 39-44.

[9] J. Wang, L. Feng, W.W. Xue. Research on server and cluster system saving energy technology. Software, 2011(2): 35-38.

[10] L.J. Gu, F.Q. Zhou, H. Meng. Study of our data center energy consumption and energy efficiency. China energy, 2010(11): 69-71.

[11] Q.T. Guo, Q.Q. Sun, Y.P. Li, X.J. Yu. Research and implementation of high performance network server framework. Computer engineering, 2013(12): 75-78.

[12] R.G. Li, J. Wang. The application of MySQL database in automatic test system. Computer application, 2011(S2): 96-99.

[13] Z.C. Wu. Application of MySQL database in small and medium sized business system. Digital technology and application, 2011(11): 52-55

[14] X.A. Qi, Q.J. Hou. Study of using SQL Server database. Manufacturing automation, 2010(14): $112-115$.

[15] X.l. Ma, G.Q. Zhao, Y.T. Zhu. The technology realization of knowledge visualization and study progress visualization in LMS. China education, 2013(1): 87-90. 